\title{
Sensor Fault Diagnosis in State Feedback Systems using Artificial Neural Networks
}

\author{
V.Manikandan', K.Ramakrishnan ${ }^{2}$, N.Devarajan ${ }^{3}$, C.K.Babu ${ }^{4}$, R.VenkatateswaraBhupathi ${ }^{5}$ \\ ${ }^{1.4}$ Dept. of Electrical and Electronics Engineering, Coimbatore Institute of Technology, Coimbatore-641 014. \\ ${ }^{2}$ Dept. of Electrical and Electronics Engineering, Pondichery Engineering College, Pondichery-605014. \\ ${ }^{3}$ Dept. of Electrical and Electronics Engineering, Government College of Technology, Coimbatore-641 013. \\ ${ }^{5}$ Dept. of Computer Science and Engineering, VLB Janakiammal College of Engineering and Technology, \\ Coimbatore-641042. \\ E-mail:babu.ck05@gmail.com, mani_sr2006@yahoo.co.in, ramss_k@yahoo.co.in
}

\begin{abstract}
A simulation before test method for fault diagnosis and classification towards sensor fault in linear time invariant state feed back system is presented in this paper. The novelty of the approach lies in associating with each state feedback gain factor a scalar , which is defined as the sensor healthiness factor. This scalar is made to vary from 1 (no fault condition) to 0 (full fault condition) in predetermined steps. The intermediate values of portray the deterioration modes of the sensor. The Integral Square Error (ISE) criterion is employed for extracting the signature of the fault and the classification is done using Artificial Neural Network (ANN) classifier. The proposed diagnosis approach is applied to a dc motor system to validate the effectiveness of the technique.program inspections, static \& dynamic analysis and V\&V techniques
\end{abstract}

Key words : Neural Network, Integral square error, fault diagnosis

\section{INTRODUCTION}

Fault detection techniques are mostly based on construction of a model of the system (Isermann et al., 1997).A second way is to constructan observer capable of estimating the trends some internal variables of the process (Patton et al., 1997). Alternatively, an estimate of some process parameters can be carried out (Hofling et al., 1994). Whichever method is used, the estimated values must be compared with the actual ones in order to obtain information on the state of the system, and eventually detect the occurrence of a fault (Rizzo et al., 2002). Recent work on fault detection tends to deal with the intrinsic nonlinear nature of systems, introducing nonlinear tools for modeling and fault detection, especially those based on soft computing, which allows both expert knowledge stored in the input-output data (Fortuna et al., 2001) to be exploited. Neural networks are generally used to build nonlinear models or nonlinear observers, thus substituting their linear correspondent in previous approaches. Significant work has been carried out recently by adopting this strategy (Polycarpou et al., June 1995, Borairi etal., July 1996, Alessandri etal., June1997, Vemuri etal., April 1998, Demetriou etal., Nov 1998 and Maki etal., Nov 1997, Marcu etal., Oct 1997, Nauck etal., 1997). The general structure of the fault diagnosis system is shown in the Fig.1. (Toscano et al., 2003). There are basically two levels system level or level 0 , whose role is mainly to generate control law in order to ensure correctperformance of the closed loop system and a supervision level or level 1 , whose role is decision making from the information generated by level 0 . The level 1 consists of an Observation function, , a Classification function, and a decision making function, . The role of the observation function is to generate, from the measures provided by the level 0 , a signature, $x$ allowing us to characterize the possible faults, which might occur on the equipment. The signature $x$ generated by the observation function is then applied to the classification function, which will allow the recognition of operating modes of the process.

The decision making function, allows us to acton the level 0 in accordance with the operating modes recognized by the classification function. Itcould be parametric $(\mathrm{K})$ adaptation of the control law to preserve the performance of the supervised system or modification of the system operating point in order to meet the production objectives or an emergency stop procedure if the operating point is hazardous to human operator/equipment.

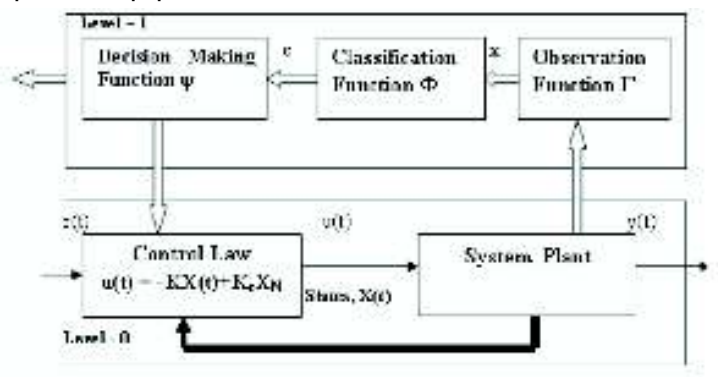

Fig.1. General fault diagnosis system

\section{PROPOSED DIAGNOSIS APPROACH}

Consider the LTI Single Input Single Output (SISO) systemdescribed by

$$
\begin{aligned}
& \stackrel{\mathrm{X}}{\mathrm{X}}(\mathrm{t})=\mathrm{AX}(\mathrm{t})-\mathrm{Bu}(\mathrm{t}) \\
& \mathrm{y}(\mathrm{t})=\mathrm{CX}(\mathrm{t})
\end{aligned}
$$


Where $A R^{n \times n}$ and is the system matrix, $B R^{n \times 1}$ is the input matrix and $C R^{1 \times n}$ is the output matrix. The state vector $X(t)$ or simply $X$ is an $n$ vector; $u(t)$ is the control effort and $y(t)$ is the system output. If the pair $(A, B)$ is controllable i.e., if the following condition is met,

$$
\operatorname{rank}\left[\begin{array}{lllll}
B & A B & A^{2} B & \ldots & A^{r} B
\end{array}\right]=n
$$

Then state feedback control for arbitrary pole placement is possible. Assuming that all the state variables are available for feedback, the control effort is given by

$$
u(t)=-K X(t)+k_{e} X_{H}
$$

Where

$$
x_{N}=\int e(t) d t
$$

Here $e(t)$ is $r(t)-y(t)$, where $r(t)$ is the reference input and $y(t)$ is the output; $K$ the state feedback gain factor given by

$$
\mathrm{K}=\left[\begin{array}{llllll}
\mathrm{K}_{1} & \mathrm{~K}_{2} & \mathrm{~K}_{1} & . & \mathrm{K}_{\mathrm{a}}
\end{array}\right]
$$

where $K_{i} s$ are the feedback gain factors. The matrix $\mathrm{K}$ can be designed either from the closed loop response specifications or optimally by LQR approach (Franklin et al., 2002). The first portion of the control law (3) basically means that each state of the system say $X_{i}$ is multiplied with the element $K_{i}$ of $K$ and summed up (i from 1 to $n$ for $n$ states) and fed back. This portion of the control law characterizes the transient performance of the closed loop system and the integral portion ensures that there is no offset in the steady state response in tracking $r(t)$. The control law is given explicitly as

$$
u(t)=-\sum_{i=1}^{n}\left[K_{i} X_{i}(t)\right]+k_{e} \int \alpha(t) d t
$$

Since only sensors provide the state of the system and that these states are feedback with a gain of $K$, to analyze the faulty and deterioration modes of the sensor, we associate a scalar for each element of the feedback matrix (which in turn means that to each sensor we associate this scalar, as the performance indicative factor). That is, for diagnosing the quality of the sensor $S_{i}$ (say a tachogenerator) that is giving $X_{i}$ (say speed, a state of the system), we associate the scalar, with the corresponding state feedback gain factor, $\mathrm{K}_{\mathrm{i}}$. In other words, we have fixed ${ }_{i}$ as the performance index for $\mathrm{S}_{i}$. This scalar is known as the Sensor healthiness factor. This factor can vary in any steps from 1 to 0 . A value of 1 for this factor means that the particular sensor is healthy and that it is transduting the actual quantity of the measurand. A value of zero means that the sensor has worn out completely and is not giving any output at all. Intermediate values portray the deterioration modes of the sensor.

The control law can now be written in the expanded form as

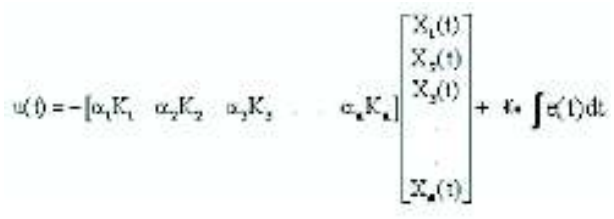

Or

$$
u(t)=-\alpha k x(t)+k_{\sigma} \int \alpha(t) d t
$$

Where $v_{0}=\left[\begin{array}{llll}v_{1} & \alpha_{2} & \ldots & \alpha_{f}\end{array}\right]^{\mathrm{r}}$ is the vector of the healthiness factors of all the sensors of the system. Now the closed loop equations of the system are given as:

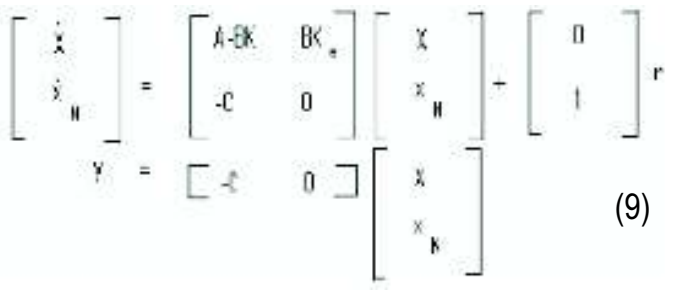

\section{DESIGNING THE NEURAL NETWORK}

Designing the neural network shown in Fig.2 involved choosing the following parameters: Number of inputs, number of output, number of hidden layers, and number of nodes/layers (Pradhan et al., 2005). In our neural network design, specifications of the above parameters were as follows:

i) Inputs $=2$

The number of inputs we fed into the system simultaneously, which was 2 for this design, these samples defined a particular pattern and was the basis of training neural network so that it could deliver desired results.

\section{ii) Outputs $=2$}

The number of the output node was just 'two' because the only output needed was ${ }_{1}$ and ${ }_{2}$.These two output conditions could be managed with two nodes. 
iii) Hidden layer $=1$

Hidden Layer Nodes $=8$

The above two values were chosen on a hit and trial basis depending on the certain performance criteria, that is goal=1e-10. "Goal" here stands healthiness factor of the sensors. One reason to choose the BP technique was the ability to change the values of its weight in response to error.

? Training data . input set \& corresponding output set

? Input. hidden layer.output

? Finds error . Output(target)-output(actual)

The network passed the derivative of the error back to the hidden layer, using an original weighted connection. Each hidden node then calculated the weighted sum of the back propagated errors to find its contribution to the known output errors. After each output layer and hidden node found the contribution, the node adjusted its weight to reduce the error.

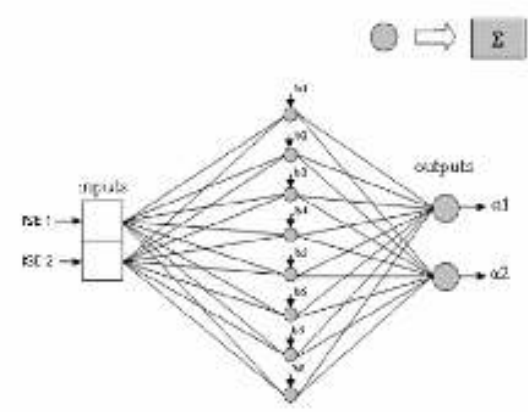

Fig.2. Designing the neural network

\section{EXTRACTION OF FAULT SIGNATURES}

The schematic for estimating the fault residue is shown in the Fig.3.The fault residue extraction process assumes that all the states are available for the feedback. Unit step is used as the input excitation. Now the sensor healthiness factor of any one sensor (or more in case we are interested in multiple fault analysis) is varied in predetermined steps between 1 thro 0 and ISE of the error $\left(X X_{f}\right)$ where $X$ is the state dynamics of the system without fault and $X_{f}$ is the state dynamics with fault is computed as the fault signature. Similar signatures for different sensors (individually or in combined fashion) are obtained and stored in a database for training the ANN Classifier. To simulate the actual working condition in this model based fault diagnosis approach, the system and control parameters are kept at their nominal values. By this, we enrich the database with a number of patterns conforming to a specific system operating condition thereby simplifying the classification process substantially.

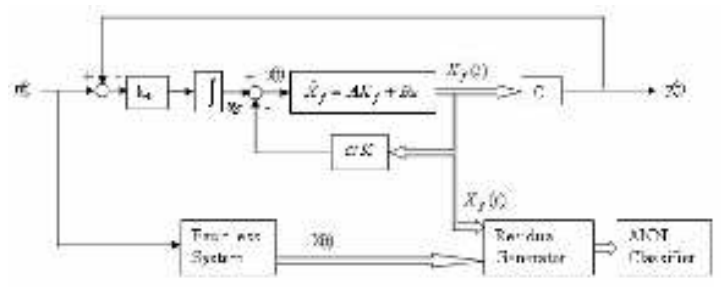

Fig.3. Extraction of fault signature

\section{RESULTS ON A DC MOTOR SYSTEM}

For a dc motor system with permanent magnet, the system descriptive equations (Katshiko Ogata, 2003) are:

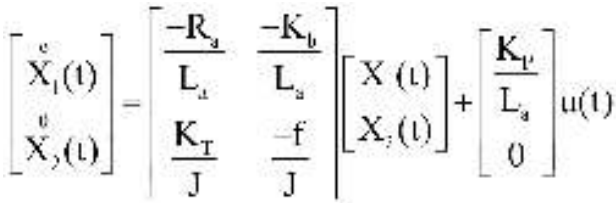

$$
\begin{aligned}
& y(t)=\left[\begin{array}{ll}
0 & 1
\end{array}\right]\left[\begin{array}{l}
X_{1}(1) \\
X_{2}(t)
\end{array}\right]
\end{aligned}
$$

Where

$X_{1}(t)$ is the Armature Current in Amps.

$\mathrm{X}_{2}(\mathrm{t})$ is the rotor speed in rad $/ \mathrm{sec}^{2}$.

$\mathrm{R}_{\mathrm{a}}$ is the armature resistance in Ohms

$L_{a}$ is the Armature inductance in Henries

$\mathrm{J}$ is the moment of inertia of the load in $\mathrm{Kg} . \mathrm{m}^{2}$

fis the viscous friction coefficient in $\mathrm{Nm} / \mathrm{rad} / \mathrm{sec}$.

$\mathrm{K}_{\mathrm{b}}$ is the back emf constant in V/rad/sec.

$\mathrm{K}_{\mathrm{T}}$ is the Torque constant.

$K_{p}$ is the Power interface gain.

For system parameters: $R_{a}=1, L_{a}=0.095 H, \quad J=0.02105$ $\mathrm{Kg} \cdot \mathrm{m}^{2}, \mathrm{f}=0.01 \mathrm{Nm} / \mathrm{rad} / \mathrm{sec}, \mathrm{K}_{b}=0.02 \mathrm{~V} / \mathrm{rad} / \mathrm{sec}, \mathrm{K}_{\mathrm{T}}=0.1, \mathrm{~K}_{\mathrm{P}}$ $=20$, the system equations are given by

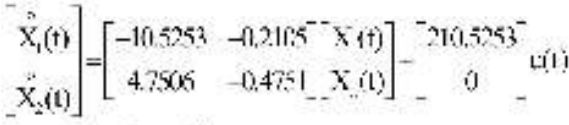

$$
\begin{aligned}
& \left.y(t)=\left[\begin{array}{ll}
0 & 1
\end{array}\right] \begin{array}{l}
X(t) \\
X_{X}(t)
\end{array}\right]
\end{aligned}
$$


The system open loop poles are located at 10.4248 and 0.5756 . The open loop system is stable but heavily over damped. The state feedback control is designed to give a closed loop response of $5 \%$ peak overshoot and 0.1 sec peak time to unit step forcing function. These specifications give locations for closed loop poles at $30.9 j 31.4159$ and third non-dominant closed loop pole is placed at 200. The control effort $u(t)$ is given by

$\left.u(t)=-1.1913 X_{1}(t)-14.1799 X_{v}(t)+388.306 x i\right] \sigma(t) d t$

\section{CASE STUDY}

Now let us make one case study wherein the current sensor has deteriorated and is giving only $50 \%$ of the actual current $\left(_{1}=0.5\right.$ and ${ }_{2}=1$ ). Now the state feedback matrix is

$$
K=\left[\begin{array}{ll}
0.5 \mathrm{~K}_{1} & \mathrm{~K}_{2}
\end{array}\right]
$$

Now ISE of $\left(X-X_{t}\right)$ give the signature for this fault condition (Table I). The waveforms for $X, X_{f}, X-X_{f}$, for $50 \%$ fault in sensor are shown in Fig.4.Similar signatures are extracted for both the sensor (current as well as speed) under fault mode. The database will be as shown in the Table II. In the Table II, we have shown that the healthiness factor of the current sensor varies from 0.5 to 0.95 of its optimum value and for each value of ${ }_{1}$, the other sensor varied from 0.5 to 1 with system and control parameters kept at their nominal values. It is quite evident that the error reduces as the quality (depicted by the healthiness factor ) of transduction of a sensor improves. All the signatures stored in the database are used for training the ANN whose effectiveness in classifying unknown pattern belonging to an appropriate class is well known and can be found through pattern classifier below.

Table 1. Integral Square Error for [0.5 1] fault condition

\begin{tabular}{|c|c|c|c|c}
\multicolumn{2}{|c|}{ Sensor } & \multicolumn{2}{|c|}{ Integral Square } & \multirow{2}{*}{ Fault } \\
Healthiness & \multicolumn{2}{|c|}{ Error(ISE) } & \multirow{2}{*}{ ID } \\
Factor & \multicolumn{2}{|l|}{} \\
\hline$\alpha_{1}$ & $\alpha_{2}$ & Current & Speed & \\
\hline 0.5 & 1 & 4.624 & 0.049 & 101 \\
\hline
\end{tabular}
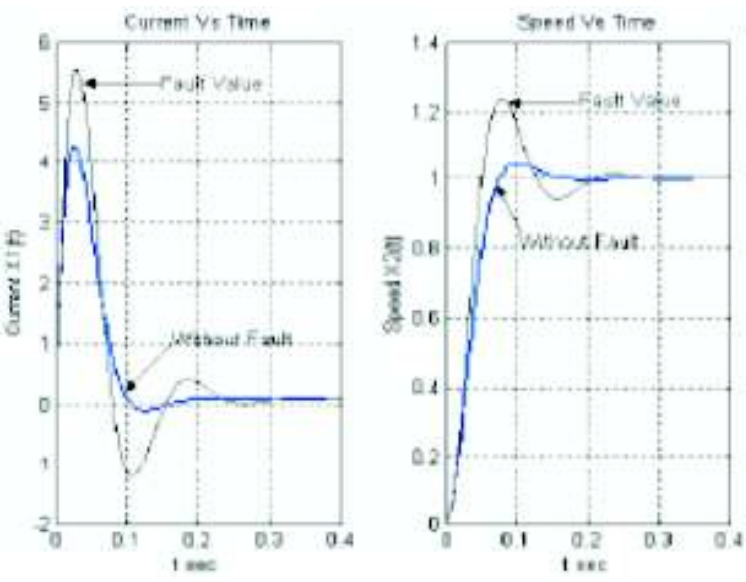

Fig. 4. a System states - current and speed - vs Time
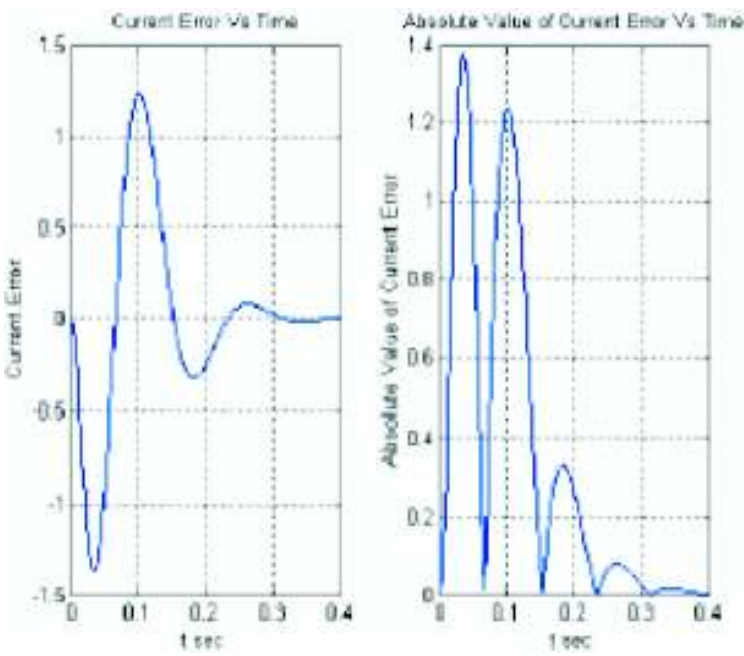

Fig. 4. B Error dynamics for current
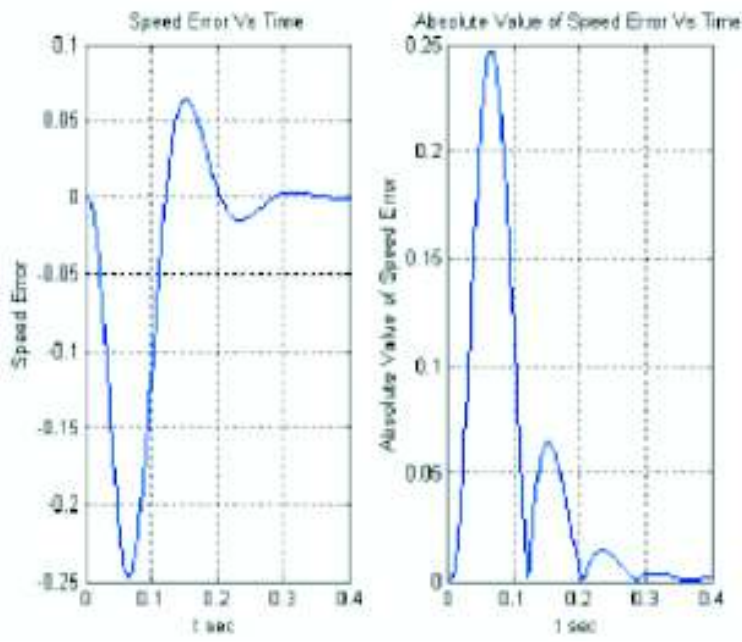

Fig. 4. C Error dynamics for Speed 
Table 2. Integral Square Error (ISE) for other fault condition

\begin{tabular}{|c|c|c|c|c|}
\hline \multicolumn{2}{|c|}{$\begin{array}{c}\text { Sensor } \\
\text { Healthiness } \\
\text { Factor }\end{array}$} & \multicolumn{2}{|c|}{$\begin{array}{c}\text { Integral Square } \\
\text { Error(ISE) }\end{array}$} & \multirow[t]{2}{*}{ Fault ID } \\
\hline$\alpha_{1}$ & $\alpha_{2}$ & Current & Speed & \\
\hline 0.5 & 0.5 & 55.470 & 0.628 & 1 \\
\hline 0.5 & 0.55 & 37.737 & 0.449 & 2 \\
\hline 0.5 & 0.6 & 26.598 & 0.326 & 3 \\
\hline 0.5 & 0.65 & 19.294 & 0.239 & 4 \\
\hline 0.5 & 0.7 & 14.362 & 0.176 & 5 \\
\hline 0.5 & 0.75 & 10.969 & 0.13 & 6 \\
\hline 0.5 & 0.8 & 8.614 & 0.097 & 7 \\
\hline 0.5 & 0.85 & 6.978 & 0.073 & 8 \\
\hline 0.5 & 0.9 & 5.854 & 0.059 & 9 \\
\hline 0.5 & 0.95 & 5.103 & 0.051 & 10 \\
\hline 0.55 & 0.5 & 48.404 & 0.611 & 11 \\
\hline 0.55 & 0.55 & 32.822 & 0.436 & 12 \\
\hline 0.55 & 0.6 & 22.928 & 0.315 & 13 \\
\hline 0.55 & 0.65 & 16.41 & 0.229 & 14 \\
\hline 0.55 & 0.7 & 12.01 & 0.166 & 15 \\
\hline 0.55 & 0.75 & 8.998 & 0.12 & 16 \\
\hline 0.55 & 0.8 & 6.927 & 0.087 & 17 \\
\hline 0.55 & 0.85 & 5.513 & 0.064 & 18 \\
\hline 0.55 & 0.9 & 4.566 & 0.049 & 19 \\
\hline 0.55 & 0.95 & 3.958 & 0.042 & 20 \\
\hline
\end{tabular}

\begin{tabular}{|c|c|c|c|c|}
\hline 0.6 & 0.5 & 43.353 & 0.603 & 21 \\
\hline 0.6 & 0.55 & 29.216 & 0.428 & 22 \\
\hline 0.6 & 0.6 & 20.175 & 0.307 & 23 \\
\hline 0.6 & 0.65 & 14.204 & 0.22 & 24 \\
\hline 0.6 & 0.7 & 10.18 & 0.157 & 25 \\
\hline 0.6 & 0.75 & 7.441 & 0.111 & 26 \\
\hline 0.6 & 0.8 & 5.578 & 0.078 & 27 \\
\hline 0.6 & 0.85 & 4.326 & 0.055 & 28 \\
\hline 0.6 & 0.9 & 3.512 & 0.041 & 29 \\
\hline 0.6 & 0.95 & 3.014 & 0.034 & 30 \\
\hline 0.65 & 0.5 & 39.679 & 0.600 & 31 \\
\hline 0.65 & 0.55 & 26.541 & 0.425 & 32 \\
\hline 0.65 & 0.6 & 18.095 & 0.303 & 33 \\
\hline 0.65 & 0.65 & 12.509 & 0.214 & 34 \\
\hline 0.65 & 0.7 & 8.752 & 0.151 & 35 \\
\hline 0.65 & 0.75 & 6.209 & 0.104 & 36 \\
\hline 0.65 & 0.8 & 4.497 & 0.071 & 37 \\
\hline 0.65 & 0.85 & 3.366 & 0.048 & 38 \\
\hline 0.65 & 0.9 & 2.65 & 0.033 & 39 \\
\hline 0.65 & 0.95 & 2.236 & 0.026 & 40 \\
\hline 0.7 & 0.5 & 37.013 & 0.603 & 41 \\
\hline 0.7 & 0.55 & 24.573 & 0.425 & 42 \\
\hline 0.7 & 0.6 & 16.541 & 0.301 & 43 \\
\hline 0.7 & 0.65 & 11.227 & 0.211 & 44 \\
\hline 0.7 & 0.7 & 7.652 & 0.146 & 45 \\
\hline 0.7 & 0.75 & 5.247 & 0.099 & 46 \\
\hline
\end{tabular}


V.Manikandan et al : Sensor Fault Diagnosis in State Feedback Systems using Artificial Neural Networks

\begin{tabular}{|c|c|c|c|c|}
\hline 0.7 & 0.8 & 3.641 & 0.065 & 47 \\
\hline 0.7 & 0.85 & 2.597 & 0.042 & 48 \\
\hline 0.7 & 0.9 & 1.953 & 0.027 & 49 \\
\hline 0.7 & 0.95 & 1.599 & 0.02 & 50 \\
\hline 0.75 & 0.5 & 35.124 & 0.612 & 51 \\
\hline 0.75 & 0.55 & 23.166 & 0.43 & 52 \\
\hline 0.75 & 0.6 & 15.415 & 0.302 & 53 \\
\hline 0.75 & 0.65 & 10.276 & 0.21 & 54 \\
\hline 0.75 & 0.7 & 6.829 & 0.143 & 55 \\
\hline 0.75 & 0.75 & 4.515 & 0.095 & 56 \\
\hline 0.75 & 0.8 & 2.981 & 0.060 & 57 \\
\hline 0.75 & 0.85 & 1.995 & 0.037 & 58 \\
\hline 0.75 & 0.9 & 1.4 & 0.022 & 59 \\
\hline 0.75 & 0.95 & 1.09 & 0.014 & 60 \\
\hline 0.8 & 0.5 & 33.856 & 0.63 & 61 \\
\hline 0.8 & 0.55 & 22.219 & 0.438 & 62 \\
\hline 0.8 & 0.6 & 14.648 & 0.306 & 63 \\
\hline 0.8 & 0.65 & 9.619 & 0.217 & 64 \\
\hline 0.8 & 0.7 & 6.245 & 0.143 & 65 \\
\hline 0.8 & 0.75 & 3.985 & 0.093 & 66 \\
\hline 0.8 & 0.8 & 2.493 & 0.057 & 67 \\
\hline 0.8 & 0.85 & 1.542 & 0.033 & 68 \\
\hline 0.8 & 0.9 & 0.978 & 0.018 & 69 \\
\hline 0.8 & 0.95 & 0.695 & 0.01 & 70 \\
\hline 0.85 & 0.5 & 33.098 & 0.643 & 71 \\
\hline 0.85 & 0.55 & 21.661 & 0.45 & 72 \\
\hline
\end{tabular}

\begin{tabular}{|c|c|c|c|c|}
\hline 0.85 & 0.6 & 14.189 & 0.313 & 73 \\
\hline 0.85 & 0.65 & 9.215 & 0.216 & 74 \\
\hline 0.85 & 0.7 & 5.874 & 0.144 & 75 \\
\hline 0.85 & 0.75 & 3.636 & 0.093 & 76 \\
\hline 0.85 & 0.8 & 2.162 & 0.056 & 77 \\
\hline 0.85 & 0.85 & 1.225 & 0.031 & 78 \\
\hline 0.85 & 0.9 & 0.675 & 0.015 & 79 \\
\hline 0.85 & 0.95 & 0.404 & 0.006 & 80 \\
\hline 0.9 & 0.5 & 32.77 & 0.665 & 81 \\
\hline 0.9 & 0.55 & 21.435 & 0.465 & 82 \\
\hline 0.9 & 0.6 & 13.999 & 0.324 & 83 \\
\hline 0.9 & 0.65 & 9.033 & 0.222 & 84 \\
\hline 0.9 & 0.7 & 5.691 & 0.148 & 85 \\
\hline 0.9 & 0.75 & 3.449 & 0.094 & 86 \\
\hline 0.9 & 0.8 & 1.971 & 0.056 & 87 \\
\hline 0.9 & 0.85 & 1.032 & 0.03 & 88 \\
\hline 0.9 & 0.9 & 0.481 & 0.013 & 89 \\
\hline 0.9 & 0.95 & 0.211 & 0.004 & 90 \\
\hline 0.95 & 0.5 & 32.807 & 0.693 & 91 \\
\hline 0.95 & 0.55 & 21.497 & 0.484 & 92 \\
\hline 0.95 & 0.6 & 14.046 & 0.337 & 93 \\
\hline 0.95 & 0.65 & 9.051 & 0.231 & 94 \\
\hline 0.95 & 0.7 & 5.678 & 0.154 & 95 \\
\hline 0.95 & 0.75 & 3.409 & 0.098 & 96 \\
\hline 0.95 & 0.8 & 1.908 & 0.058 & 97 \\
\hline 0.95 & 0.85 & 0.952 & 0.03 & 98 \\
\hline
\end{tabular}




\begin{tabular}{c|c|c|c|c|}
0.95 & 0.9 & 0.387 & 0.013 & 99 \\
\hline 0.95 & 0.95 & 0.107 & 0.003 & 100 \\
\hline 0.5 & 1 & 4.624 & 0.049 & 101 \\
\hline 0.55 & 1 & 3.601 & 0.04 & 102 \\
\hline 0.6 & 1 & 2.749 & 0.032 & 103 \\
\hline 0.65 & 1 & 2.041 & 0.025 & 104 \\
\hline 0.7 & 1 & 1.458 & 0.019 & 105 \\
\hline 0.75 & 1 & 0.986 & 0.013 & 106 \\
\hline 0.8 & 1 & 0.616 & 0.009 & 107 \\
\hline 0.85 & 1 & 0.338 & 0.005 & 108 \\
\hline 0.9 & 1 & 0.147 & 0.002 & 109 \\
\hline 0.95 & 1 & 0.036 & 0.001 & 110 \\
\hline 1 & 1 & 0 & 0 & 111 \\
\hline
\end{tabular}

\section{PATTERN CLASSIFIER}

Since the test pattern for the $D C$ motor system has two controlling inputs that is ANN has 2 neurons in the input layer and 2 neurons in the output layer. The 2 neurons in the output layer can classify all types of faults and will be sufficient for classifying total of 111 different faults. The number of neurons in the hidden single layer is 8. So the ANN structure boils down to 2:8:2. The pattern for a specific fault is generated by testing the system at all test conditions under permissible tolerances for other types of faults. The ANN is adaptively trained to update the weights and the bias by gradient descent method by the mean square error performance. The classifier structure for the circuit and the training pattern for 100 epochs are shown in the Fig.5. and Fig.6. respectively. For few randomly generated test patterns for the system, classifier results are shown in the Table III. The results agree well within the corresponding fault ID (Nauck et al., 1997).

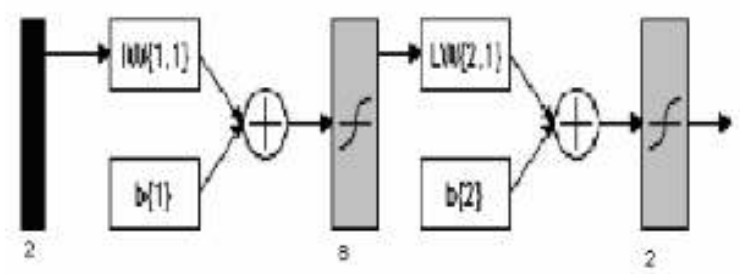

Fig. 5. Classifier for test circuit

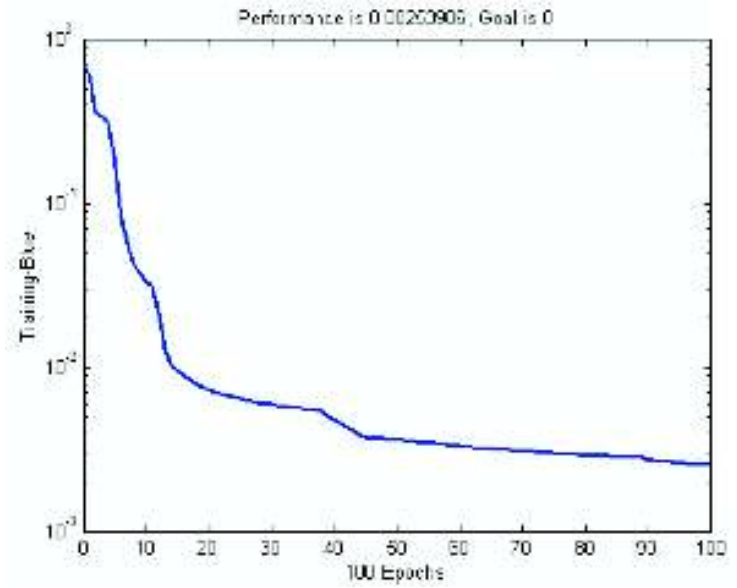

Fig. 6. Training pattern

Table 3. Few Results of Pattern classifier

\begin{tabular}{|c|c|c|c|c|}
\hline \multicolumn{2}{|c|}{ Classifier input } & \multicolumn{2}{|c|}{$\begin{array}{l}\text { Classifier } \\
\text { ouiput }\end{array}$} & \multirow{3}{*}{ Fault id } \\
\hline Integral sq & re error & \multirow{2}{*}{$\alpha_{1}$} & \multirow{2}{*}{$\alpha_{2}$} & \\
\hline current & speed & & & \\
\hline 2.597 & 0.042 & 0.7 & 0.85 & 48 \\
\hline 37.737 & 0.449 & 0.5 & 0.55 & 2 \\
\hline 15.415 & 0.302 & 0.75 & 0.6 & 53 \\
\hline 43.353 & 0.603 & 0.6 & 0.5 & 21 \\
\hline 33.856 & 0.63 & 0.8 & 0.5 & 61 \\
\hline 4.624 & 0.049 & 0.5 & 1 & 101 \\
\hline 0.387 & 0.013 & 0.95 & 0.9 & 99 \\
\hline 12.01 & 0.166 & 0.55 & 0.7 & 15 \\
\hline
\end{tabular}




\begin{tabular}{c|c|c|c|c}
0 & 0 & 1 & 1 & 111 \\
14.189 & 0.313 & 0.85 & 0.6 & 73 \\
\hline 6.245 & 0.143 & 0.8 & 0.7 & 65 \\
\hline 14.046 & 0.337 & 0.95 & 0.6 & 93 \\
\hline 5.578 & 0.078 & 0.6 & 0.8 & 27 \\
\hline 55.470 & 0.628 & 0.5 & 0.5 & 1 \\
\hline
\end{tabular}

\section{CONCLUSION}

A novel Simulation before Test approach towards sensor fault diagnosis in full state feedback system is proposed in this paper. Associating a sensor healthiness factor with each element of the feedback gain matrix and varying them from 1 thro 0 , deteriorating modes of the sensors are analyzed. The performance criterion Integral Square Error (ISE) is used for fault signature extraction and ANN is employed for fault classification. The proposed approach for single sensor fault is illustrated through a dc motor system example with encouraging results.

\section{REFERENCES}

[1] Alessandri A and Parisini T, June 1997 "Model based fault diagnosis using non linear estimators: A neural approach," in Proc.Amer. Contr. Conf. vol. 15, pp. 2874-2878.

[2] Borairi $M$ and Wang $H$, July 1996, "Actuator and sensor fault diagnosis of non linear dynamic systems via genetic neural networks and parameter estimation techniques," in Proc.IEEE Int .Conf.Contr.Applicat., vol.1, pp. 278-282.

[3] Demetriou M A and Polycarpou M M, Nov.1998, "Incipient fault diagnosis of dynamical systems using on line approximations," IEEE trans. Automat. Control, vol 43, pp.1612-1617.

[4] Fortuna L, Rizzotto G G, Lavorgna M, Nunnari G, Xibilia M G and Caponetto R, 2001, "Soft computing new trends and Applications," in advanced textbooks in control and signal processing. London, U.K., Springer Verlog.

[5] Franklin F Gene, David Powel J, Emami-Naeni A, 2002, 'Feedback Control of Dynamic Systems', Fourth Edition, Pearson Ed.
[6] Hofling T and Feufer TP, 1994, "Detection of additive and multiplicative faults-parity space vs. parameter estimation," in Proc .IFAC SAFEPROCESS Symp., Baden-Baden,Germany.

[7] Isermann R and Balle P, 1997, "Trends in application of model based fault detection and diagnosis of technical processes," Control Eng. Practice, vol.5, no.5, pp.709-719.

[8] Katshiko Ogata, 2003, 'Modern Control Engineering', Third Edition, $\mathrm{PHI}$.

[9] Maki Y and Loparo KA, Nov1997, "A neural network approach to fault diagnosis in industrial processes," IEEE Trans. Contr. syst. Technol., vol.5, pp.529541.

[10] Marcu T and Mirea L, Oct1997, "Robust detection and isolation of process faults using neural networks," IEEE Contr. Syst. Mag., vol.17, no.5, pp. 72-79.

[11] Nauck D and Kruse R, 1997, 'ANeuro-Fuzzy Method to learn Fuzzy Classification Rules from Data', Fuzzy Sets and Systems 89, pp. 277-288.

[12] Patton R J and Chen J, 1997, "Observer based fault detection and isolation: Robustness and applications", Control Eng. Practice, vol.5, no.5, pp.671-682.

[13] Polycarpou M M and Venmuri A T, June 1995, "Learning methodology for failure detection and accommodation," IEEE Control System Magazine., Pp.16-24.

[14] Pradhan A K et al, 2005, "Implementation of an intelligent relay using nios processor," Nios soft core embedded processor design contest outstanding project collections, pp. 38-45.

[15] Rizzo Alessandro and Gabriella Xibilia M, May 2002, "An innovative intelligent system for sensor validation in tokomak machines," IEEE Trans.Control system technology, vol.10, no.3.

[16] Toscano R and Lyonnet P, 2003, 'Diagnosis of Industrial Systems by Fuzzy Classifier', ISA Transactions 42, pp.327-335.

[17] Vemuri A T, Polycarpou M M and Diakourtis S A, April 1998, "Neural network based fault detection in robotic manipulators, "IEEE trans. Robot Automat. Contr., vol.14, pp. 342-348.

[18] MATLAB 6p5 Source Codes. 